\title{
Médiévales
}

Langues, Textes, Histoire

67 | automne 2014

Histoires de Bohême

\section{Emeri VAN DONZEL et Andrea SCHMIDT, Gog and Magog in Early Eastern Christian and Islamic Sources. Sallam's Quest for Alexander Wall}

Leyde, Brill, 2010, $271 \mathrm{p}$.

\section{Anna Caiozzo}

\section{OpenEdition}

\section{Journals}

Édition électronique

URL : https://journals.openedition.org/medievales/7325

DOI : 10.4000/medievales.7325

ISSN : $1777-5892$

\section{Éditeur}

Presses universitaires de Vincennes

Édition imprimée

Date de publication : 31 décembre 2014

Pagination : 187-189

ISBN : 978-2-84292-422-5

ISSN : 0751-2708

\section{Référence électronique}

Anna Caiozzo, «Emeri van donzel et Andrea schmidt, Gog and Magog in Early Eastern Christian and Islamic Sources. Sallam's Quest for Alexander Wall », Médiévales [En ligne], 67 | automne 2014, mis en ligne le 09 mars 2015, consulté le 23 avril 2022. URL : http://journals.openedition.org/medievales/7325 ; DOI https://doi.org/10.4000/medievales.7325

Ce document a été généré automatiquement le 23 avril 2022

Tous droits réservés 


\section{Emeri VAN DONZEL et Andrea SCHMIDT, Gog and Magog in Early Eastern Christian and Islamic Sources. Sallam's Quest for Alexander Wall}

Leyde, Brill, 2010, 271 p.

Anna Caiozzo

\section{RÉFÉRENCE}

Emeri VAN DONZEL et Andrea SCHMIDT, Gog and Magog in Early Eastern Christian and Islamic Sources. Sallam's Quest for Alexander Wall, Leyde, Brill, 2010, 271 p.

1 L'enquête sur la localisation du fameux mur des Gog et Magog, ces peuples cités par la Bible dans le Livre d'Ézéchiel, et par le Coran dans la sourate XVIII, a été menée par bon nombre de chercheurs. Parmi les études phares, et sans compter toutes celles dédiées à Alexandre, on peut citer pour commencer celles d'un précurseur, A. Runni Anderson, "Alexander and the Caspian Gates", Transactions and Proceedings of the American Philological Association, 59 (1928), p. 130-163, et Alexander's Gate, Gog and Magog and the Inclosed Nations, Cambridge, 1932. Rappelons encore celle de Paolo Cuneo, «Le mura di Darbent, Note sulla topografia e la morfoligia urana di una città-stato del limes islamico nell'area caucasica ", Rivista degli Studi orientali, p. 57-75; et, plus récemment, l'article de Charles Genequand, "Autour de la ville de Bronze: d'Alexandre à Salomon", Arabica, 1992, p. 328-345; ou encore les notes d'André Miquel dans La Géographie humaine du monde musulman jusqu'au milieu du XI $\mathrm{X}^{e}$ siècle, vol. 2, Paris, 1975 réimp. Paris, EHESS, 2001, p. 490-493. Bref, la muraille est un topos littéraire et historiographique qui a donné lieu à quelques belles études et qui, sans aucun doute, n'a pas fini d'alimenter les interrogations. 
2 Toutefois, l'enquête menée par Emeri van Donzel et Andrea Schmidt est, soulignons-le, passionnante, et d'un grand intérêt pour les chercheurs passionnés de folklore, de merveilleux, mais aussi soucieux de décoder les sources orientales, arabes, persanes, littéraires ou non. Cet ouvrage apporte un complément à celui de F. Doufikar Aerts, Alexander Magnus Arabicus: A Survey of the Alexander Tradition Through Seven Centuries: from Pseudo-Callisthenes to Suri, Peeters, 2010. Certes, comme les auteurs se plaisent à le dire, le lecteur n'aura pas une étude exhaustive de toutes les sources, mais une mise en évidence du rôle des sources syriaques à la base de la tradition islamique. Quant à la seconde partie de l'ouvrage, tout à fait capitale, elle analyse le fameux témoignage de l'envoyé du calife al-Wâthiq, le mystérieux Ibn Sallam. Une seule restriction s'appliquera à l'image de couverture : car il est assez surprenant, à l'heure où les miniatures ne sont plus considérées comme de beaux ornements mais comme des témoignages culturels de leur temps, que la référence de l'illustration de couverture, à savoir la célèbre Khamsa de Bâysunghur conservée au Topkapi Sarayi à Istanbul, le manuscrit Hazine 796, ne soit pas donnée par les auteurs. Or, la représentation de la fameuse muraille, dès le XIII ${ }^{e}$ siècle, a de quoi occuper plus d'un spécialiste de l'image ou de l'imaginaire.

La première partie est dédiée à l'analyse critique des sources sur la barrière. Le chapitre 1 évoque l'origine des deux concepts et leur étymologie depuis l'époque sumérienne, dans la Bible et la littérature rabbinique, les oracles sibyllins, Flavius Josèphe, dans la littérature chrétienne primitive. On comprend qu'ils deviennent le symbole par excellence de l'ennemi à un moment donné : Huns, Khazars, Arabes, Turcs, Mongols, et de n'importe quel agresseur d'un peuple ou d'un territoire. Leur nom rejaillit dans les milieux juifs d'Alexandrie, où l'on commence à dire qu'Alexandre le Grand avait construit une barrière contre l'ennemi dans le Nord, et où Flavius Josèphe parle, lui, du danger des Scythes (de fait, un Empire scythe avait existé du viII au $\mathrm{II}^{\mathrm{e}}$ siècle). La localisation de ce mur n'est pas définie ; on évoque souvent Darbend dans le Caucase, la porte des Alains. La littérature chrétienne (saint Jean influencé par le Livre des Jubilées et Les Oracles sibyllins) parle également de ces peuples damnés (saint Jérôme les compare aux Huns). Le chapitre 2 évoque Alexandre et Gog et Magog dans les sources orientales chrétiennes et le lien entre les diverses traditions (la tradition syriaque ayant influencé les autres), ainsi que leur ancrage dans le présent des peuples à chaque époque (Le Roman d'Alexandre du Pseudo-Callisthène). Les auteurs évoquent ensuite l'Apocalypse de Daniel (629), l'Apocalypse syriaque du Pseudo-Méthode, la chronique de Michel le Syrien (xII ${ }^{e}$ siècle), la version copte et éthiopienne de la Romance, la tradition géorgienne. Certains éléments sont récurrents : les yeux bleus, les cheveux rouges, le cannibalisme et le mur construit par Alexandre pour protéger le monde. Le chapitre 3 rappelle les liens entre Iskandar et les Gog et Magog dans le Coran. Et le chapitre 4 développe les traits de la légende dans la tradition islamique, leurs pratiques culturelles, les lieux fréquentés (problématiques), leurs caractéristiques de races monstrueuses mangeant des nourritures interdites, dont les hommes et les morts, et leurs liens avec l'eschatologie. Les sources arabes établissent également des liens entre Gog et Magog et les Turcs. Le chapitre 5 explicite la légende dans la prose arabe, persane et turque. On localise les Gog et Magog dans le Caucase, mais aussi dans le Nord-Est du monde ; le témoignage d'Ibn Rustah est fondamental, comme celui d'Ibn Hawqal, qui en font des peuples «fréquentables » du septième climat. La synthèse des thèmes communs à toutes ces sources permet de noter que leur apparence demeure en 
grande partie celle des sources chrétiennes (yeux bleus, cheveux et barbes rouges) de Mas'ûdî à Khwandamir. Une autre constante : le fameux tinnin, ou dragon marin tombé des nuages, dont une des têtes ressemble à une tête humaine, selon Ibn al-Faqîh, et qui possède des ailes et des écailles comme un poisson. Le chapitre 6 traite enfin de la barrière dans la littérature arabe des lettrés, l'adab, et littérature populaire.

4 La seconde partie de l'étude est dédiée au voyage de Sallam et à la fameuse barrière qu'il décrivit au calife al-Wâthiq. Le chapitre sept détaille cette ambassade, qui partit de Samarra, et avance des éléments sur l'identité de Sallam: était-il khazar, ce qui expliquerait sa connaissance du turc attestée par Ibn Rustah, mais comme on le crédite aussi de parler trente langues connues, il aurait pu être juif et commerçant. Le texte du voyage est édité par les auteurs ; il s'agit de la relation fournie par Ibn Khurradâdhbih, qui évoque le rêve terrifiant du calife et la volonté d'envoyer sur ces lieux un homme pour le renseigner; c'est le Turc Ashnas qui lui fournit alors la bonne personne. Le voyage prit seize mois à l'aller et douze mois au retour. Ce texte est connu de certains auteurs, tel Muqaddasî qui aurait vu l'ouvrage dans la bibliothèque de l'émir bouyide Adud al-Dawla (m. 983). Les auteurs s'étonnent également que bon nombre de compilateurs, lexicographes, géographes, ne parlent ni de Sallam ni de la fameuse barrière, surtout au $\mathrm{x}^{\mathrm{e}}$ siècle. Le chapitre 8 est dédié à l'origine de la description de Sallam qui serait en grande partie et de prime abord issue de la littérature chrétienne et syriaque à Basra ; ainsi la version syriaque du roman d'Alexandre aurait pu servir de modèle au récit de Sallam. Le chapitre 9 , aux origines du voyage, lève le voile sur les pseudo-motifs de l'enquête confiée à Sallam, le rôle du rêve dans le monde musulman comme annonciateur de faits à venir. On s'aperçoit que le calife al-Wâthiq se renseigne également sur les Dormants par une ambassade à Byzance. Le chapitre 10 lève le voile sur le trajet de Salam qui part vers le Caucase, lieu supposé de la barrière pour remonter vers le nord de la Caspienne, puis le Daghestan chez le Filan-Shah qui lui donne une lettre pour les Khazars, ensuite les chez les Bashkirts au nord de la mer d'Aral, et enfin le Kazakhstan, région où pousse l'assa-foetida, une plante nauséabonde, entre Tachkent et Samarkand. Puis il entre dans le territoire des Ghuzz ou Oghuz, et là il observe des places fortifiées avec des commerçants. Alors Sallam, qui aurait voyagé le long du bassin Dzungari, arrive au lac Balkakh et, enfin, va parcourir la route nord du Tarim, voyant bon nombre de cités ruinées, ce qu'il attribue à l'œuvre de Gog et Magog.

5 Lorsqu'on replace le voyage dans le contexte historique de l'époque, on s'aperçoit que la région du Tarim fut, aux $\mathrm{VII}^{\mathrm{e}}$-VIII ${ }^{\mathrm{e}}$ siècles, l'objet de luttes entre Chinois et Tibétains pour contrôler la Route de la soie. Par ailleurs, au début du viII ${ }^{\mathrm{e}}$ siècle, les Turcs orientaux, ou Ouighours, s'allient aux Tibétains contre les Turkesh. Enfin, au milieu du $\mathrm{VIII}^{\mathrm{e}}$ siècle, les Arabes dominent l'Asie centrale, alors qu'un siècle plus tard, vers 840, l'empire ouighour est en plein déclin (d'où les villes ruinées). Dans toutes ces cités du Tarim se sont développés manichéisme, bouddhisme et christianisme nestorien avec leurs hagiographies et leur littérature eschatologique (Sept dormants); d'ailleurs, la mosquée de Karahodja est consacrée aux Dormants. On retrouve les liens entre les Dormants, Iskandar et la Sourate XVIII, car dans ces milieux évangélisés par des Nestoriens convergent croyances chrétiennes et musulmanes et tous les mythes relatifs à Iskandar. Le chapitre 11 lève le voile sur la destination finale du voyage à proximité de la cité de Dunhuang, à savoir Yumenguan, ou Porte de Jade, dont les murailles et les portes monumentales ont été construites sous la dynastie Han (III siècle avant et après J.-C.) et dont la description est connue grâce à la Risala d'Abû Dulaf sous le Samanide 
Nasr ibn Ahmad. Le chapitre 12 évoque enfin le retour. Ainsi, le mystère est levé sur le fameux mur et les ennemis contenus. Car le lecteur, à la fin de l'enquête, se laisse convaincre que la fameuse muraille est bien à l'est du Tarim : les arguments historiques sont convaincants et c'est presque à regret que l'on abandonnerait l'hypothèse du Caucase cher aux imaginaires iraniens. En effet, le sage roi Khusraw Anûshirwan le fortifia pour le protéger des invasions car, bien avant le calife al-Wâthiq, il avait fait un rêve prémonitoire d'un animal sorti de la Caspienne qui l'avertissait du danger.

Mais, comme le montrent depuis le XIV siècle les copies enluminées du Shâh nâma de Firdawsî, le Caucase et l'Orient du monde demeurent bien dans la culture visuelle les deux lieux marquant de l'eschatologie, avec d'une part le tyran Dhahhâk cloué dans les parois d'une grotte par le héros Farîdûn dans le mont Damâwend, et d'autre part les Yâ'jûj wa-Mâjûj confinés par Alexandre. Le point commun est bien l'airain et le travail des forgerons qui manipulent les métaux et, de façon apotropaïque, permettent de placer dans tous les endroits sensibles ou dangereux, dans les lieux de passage, des objets ou des constructions humaines certes, mais d'essence magique, en somme des talismans servant à vaincre le mal: la finalité de la muraille de Gog et Magog ne se situant pas seulement dans sa localisation, mais aussi dans sa fonction symbolique et protectrice, faisant du souverain qui la construit un inspiré et un magicien, les traits de la royauté mythique idéale que l'on prête de ce fait à Iskandar. 